\title{
Rats: gnawing through the barriers to understanding genetic susceptibility and breast cancer
}

\author{
Anneke C Blackburn* \\ See related research by Smits et al., http://breast-cancer-research.com/content/13/4/R81
}

\begin{abstract}
Advances in genotyping technology have provided us with a large number of genetic loci associated with cancer susceptibility; however, our ability to understand the functional effects of the genetic variants of these loci remains limited. In the previous issue, Smits and colleagues demonstrate the use of congenic rat strains to discover that the Mcs5a breast cancer susceptibility locus is most likely acting through the immune system, via novel transcriptional regulatory mechanisms. This challenges our conventional thinking of cancer susceptibility and gene regulation pathways, and illustrates the potential for rodent models to help us functionally characterize polymorphisms of cancer-associated loci.
\end{abstract}

In this age of the genome-wide association study, our ability to identify genomic regions associated with disease has made great advances; however, our ability to translate this into a better understanding of the mechanisms of disease and genetic susceptibility is still limited. In the previous issue of Breast Cancer Research [1], the continued study of the Mcs5a locus, which was first identified in 2001 in 7,12-dimethylbenz(a)anthracene (DMBA)-treated rats and validated as relevant in humans in 2007 [2,3], has stretched our perspectives by highlighting how the functional role of a breast cancer susceptibility allele may be outside of the mammary epithelial cell, the target cell of breast carcinogenesis, and may originate outside of the mammary gland itself. The study utilizes congenic rat lines developed on a susceptible

*Correspondence: anneke.blackburn@anu.edu.au

The John Curtin School of Medical Research, Australian National University, PO Box 334, Canberra ACT 2601, Australia
(Wistar-Furth) genetic background, and carrying the selected resistant (Wistar-Kyoto) Mcs5a alleles, up to approximately $116 \mathrm{~kb}$ in size [3]. By performing transplantation experiments, Smits and colleagues [1] clearly demonstrate that the mammary carcinoma susceptibility phenotype mediated by $M c s 5 a$ is not transferable by transplantation of the mammary gland [1]. As their previous work had indicated expression of genes (Fbxo10 and Frmpd1) within the Mcs5a locus differed in immune tissues but not in mammary glands of resistant compared to susceptible rats [3], the involvement of the immune system was investigated with a bone marrow transplantation approach. While there are technical limitations to these experiments, the number of carcinomas in resistant hosts bearing marrow from susceptible donors was $60 \%$ higher than in resistant hosts bearing marrow from resistant donors, indicating that $M c s 5 a$ can act through components of the immune system to modulate mammary carcinoma susceptibility. Investigation of the immune cells of the resistant rats found an association with an overabundance of $\gamma \delta$ T-cell receptor (TCR) $+\mathrm{T}$ cells (important in mucosal surface surveillance), higher L-selectin expression (which may promote extravasation), increased mitogen-induced proliferation and increased production of Th1 (but not Th2) cytokines compared with susceptible rats [1].

While the target cell for Mcs5a originates outside the mammary gland, the phenotype is most strongly expressed within the carcinogen-exposed mammary gland. The T-cell differences were markedly greater in the $\mathrm{T}$ cells isolated from mammary ductal fragments or from the inguinal lymph nodes of rats treated with DMBA. Further, $\gamma \delta$-TCR $+\mathrm{T}$ cells of the mammary gland displayed different cell surface markers to the splenic $\gamma \delta$ TCR $+\mathrm{T}$ cells, and while $\gamma \delta$-TCR $+\mathrm{T}$ cells are the predominant $\mathrm{T}$-cell type in the mammary gland, they are a very minor population in the carcinomas arising in these rats [1]. This highlights the importance of analyzing the function of candidate genes in the appropriate tissue 
and under appropriate stress conditions, as the phenotypic differences of modifier alleles may be subtle or highly specific and therefore easily overlooked in the wrong setting. Such functional studies, especially involving the preneoplastic/susceptible normal gland, are much more readily achieved in rodent models than with human tissue [4].

Not only has the Mcs5a locus challenged the dominant paradigm that genetic susceptibility to mammary carcinomas will be due to phenotypes expressed in the mammary gland, it has also pushed our understanding of how the phenotypic differences are played out at the DNA level. In a second paper, Smits and colleagues [5] begin to elucidate the molecular mechanisms of the genetic variants. The Mcs5a locus consists of two nonprotein coding genetic elements that must be on the same chromosome to elicit the phenotype [3]. Smits and colleagues [5] identify a conserved looping structure in the DNA, where the looped elements are bound by CTCF and cohesion. This brings the polymorphic Mcs5al MCS5A 1 and 2 elements, which are separated by over 60 $\mathrm{kb}$ in the genome, physically close together to mediate their transcriptional regulatory differences, controlling the expression of Fbxo10 in T cells [5].

The definitive experiments are still to be done to prove that Fbxo10 expression in $\mathrm{T}$ cells is the pathway mediating the resistant phenotype of the Mcs5a locus. These could include examining the effect of tissue-specific disruption of Fxbo 10 in T cells on tumour development, or examination of tumour susceptibility in the congenic rat lines after depletion of $\mathrm{T}$ cells. Nonetheless, the current reports from the Gould laboratory are consistent with $M c s 5 a$ mediating its phenotype through an immune surveillance mechanism within the mammary gland. Over the past 10 years, evidence has been accumulating that cells of the immune system are required for the prevention of cancer [6], such that 'evading immune destruction' has been included as an emerging hallmark of cancer in a recent revision of the landmark paper from Hanahan and Weinberg [7].

The relevance of the Mcs5a locus to human breast cancer is clear, as MCS5A2 has recently been shown to be among the most significant of a list of 710 candidate breast cancer risk alleles, modifying risk to both hormone receptor-positive and -negative breast cancer [8]. With odds ratios of 1.19 and 0.77 and minor allele frequencies of 0.26 and 0.12 for MCS5A1 and MCS5A2, respectively, they are classified as high population frequency, low penetrance modifier alleles [3]. Smits and colleagues provide further evidence supporting the broad relevance of this locus to breast cancer by showing that the resistant congenic rats are also resistant to mammary carcinomas induced by an alternative carcinogen ( $\mathrm{N}$-nitroso- $\mathrm{N}$ methylurea, or NMU) or HER2/neu overexpression.
Interestingly, resistance to HER2/neu in situ carcinomas was not evident, indicating that Mcs5a is not acting during tumour initiation, but is acting during early mammary cancer progression [1], an observation consistent with Mcs5a mediating its phenotype through an immune surveillance mechanism.

Here we have the first evidence that this low penetrance, high frequency genetically determined breast cancer risk factor may be acting through the immune system, affecting long-range gene expression via novel gene regulatory elements involving three-dimensional looping DNA structures. The understanding of the threedimensional structure of DNA and chromatin and how it can impact on disease susceptibility takes the idea of functional testing of single nucleotide polymorphisms to a whole extra level of complexity. Adding to this that the target tissue of the polymorphism may not be the mammary gland, but could be the immune system, or other extra-mammary systems, functional investigations become expensive and complex. With our rodent helpers, we will continue to gnaw our way through these barriers to understanding the phenotypic expression of genetic variants involved in complex diseases, to fulfil the promises of novel pathways and therapeutic or preventive approaches offered by the age of the genome-wide association study.

Abbreviations

DMBA, 7,12-dimethylbenz(a)anthracene; TCR, T-cell receptor.

Competing interests

The author has no competing interests.

\section{Authors' contributions}

$A C B$ conceived and composed this editorial.

\section{Acknowledgements}

ACB is funded by the NHMRC, NBCF and Cancer Australia.

\section{Published: 12 October 2011}

\section{References}

1. Smits BMG, Sharma D, Samuelson DJ, Woditschka S, Mau B, Haag JD, Gould MN: The non-protein coding breast cancer susceptibility locus Mcs5a acts in a non-mammary cell-autonomous fashion through the immune system and modulates T-cell homeostasis and functions. Breast Cancer Res 2011, 13:R81.

2. Lan H, Kendziorski CM, Haag JD, Shepel LA, Newton MA, Gould MN: Genetic loci controlling breast cancer susceptibility in the Wistar-Kyoto rat. Genetics 2001, 157:331-339.

3. Samuelson DJ, Hesselson SE, Aperavich BA, Zan Y, Haag JD, Trentham-Dietz A, Hampton JM, Mau B, Chen KS, Baynes C, Khaw KT, Luben R, Perkins B, Shah M, Pharoah PD, Dunning AM, Easton DF, Ponder BA, Gould MN: Rat Mcs5a is a compound quantitative trait locus with orthologous human loci that associate with breast cancer risk. Proc Natl Acad Sci U S A 2007, 104:6299-6304.

4. Blackburn AC, Jerry DJ: Map making in the 21st century: charting breast cancer susceptibility pathways in rodent models. J Mammary Gland Biol Neoplasia 2011, 16:57-64.

5. Smits BMG, Traun BD, Devries TL, Tran A, Samuelson DJ, Haag JD, Gould MN: An insulator loop resides between the synthetically interacting elements of the human/rat conserved breast cancer susceptibility locus MCS5A/ Mcs5a. Nucleic Acids Res 2011 Sep 13 [Epub ahead of print]. 
6. Teng MW, Swann JB, Koebel CM, Schreiber RD, Smyth MJ: Immune-mediated dormancy: an equilibrium with cancer. J Leukoc Bio/ 2008, 84:988-993.

7. Hanahan D, Weinberg RA: Hallmarks of cancer: the next generation. Cell 2011, 144:646-674

8. Mavaddat N, Dunning AM, Ponder BA, Easton DF, Pharoah PD: Common genetic variation in candidate genes and susceptibility to subtypes of breast cancer. Cancer Epidemiol Biomarkers Prev 2009, 18:255-259.

\section{doi:10.1186/bcr2939}

Cite this article as: Blackburn AC: Rats: gnawing through the barriers to understanding genetic susceptibility and breast cancer. Breast Cancer

Research 2011, 13:112. 